\title{
Performance Enhancement of Wind Turbine
}

\author{
Swapnil Kumar
}

\begin{abstract}
Wind turbine performance and efficiency used to face big challenges due to the highly random nature of the wind and its own small size. Wind turbine blade geometry has direct implications on the load bearing response and performance of the blade. New Wind Turbine Blade was modelled and detailed analysis was done using Ansys and Matlab. Static, Fatigue, Vibration, Computational Fluid Dynamics and Simulink Analysis was done to compare the performance of both wind turbine blades. Velocity of $83.33 \mathrm{~m} / \mathrm{sec}$ have been incorporated for analysis. Various different Mathematical Equations and proper methodology was carried out to enhance the performance of Wind Turbine. Simulink Model was designed to optimize the performance of Wind Turbine. High Lift to Drag Parameter is optimized for proper Efficiency of Wind Turbine. Turbine blades are twisted so they can always present an angle that take advantages of the ideal lift-to-drag ratio. Optimization of Tower Design was carried out to enhance the performance of wind turbine. Better energy Production parameter is solved by the analysis and Simulation. Simulink Model was designed to optimize the performance of Wind Turbine. Simulink Output results shows the output of Electromagnetic Torque, Stator Current and Rotor Speed. Stress vs Strain Graph was plotted for both designed wind Turbine blades. Coefficient of drag graph was plotted to conclude the performance of Wind Turbine Blades. Turbulence behaviour is observed for both the wind turbine blades to validate the performance of Wind Turbine blades. Epoxy Material is considered for Wind Turbine blades.
\end{abstract}

Keywords: Performance, Efficiency, Damage, Blades

\section{INTRODUCTION}

This Wind turbine performance and efficiency used to face big challenges due to the highly random nature of the wind and its own small size [1]. Wind turbine blades with elongated rotor blade diameters and slender width are going to enhance the performance of wind Turbine. Wind turbine blade geometry has direct implications on the load bearing response and performance of the blade. Over the years, there have been continuous efforts made towards increasing the energy production and minimizing the dynamic loads on the turbine [2]. From small wind plants for powering farms and residences, in the 20th century; to larger utility scale wind generators that could connect to electricity grids for remote use of power; to current wind-powered generators in every sizes, between tiny plants for battery charging, up to near-gigawatt sized wind farms at the offshores for providing electricity to national electrical networks. From 1984 to until mid-1980's, the US ran a program to create a utility scale wind turbine industry to advance the technology and enable

Revised Manuscript Received on December 30, 2019.

* Correspondence Author

Swapnil Kumar, Bachelor of Technology , Mechanical Specialization in Automotive department, Vellore Institute of Technology,Vellore.

(C) The Authors. Published by Blue Eyes Intelligence Engineering and Sciences Publication (BEIESP). This is an open access article under the CC BY-NC-ND license (http://creativecommons.org/licenses/by-nc-nd/4.0/) large commercial wind turbines. [3] The NASA wind turbines were developed under the same program. This research and development program pioneered many of the multi-megawatt turbine technologies in use today, including: variable- speed generators, steel tube towers, partial-span pitch control, composite blade materials, along with aerodynamic, structural, and acoustic engineering design capabilities [4]. The large wind turbines developed under this effort set several world records for diameter and power output. The MOD2 wind turbine cluster produced 7.5 megawatts, the MOD-5B produced 3.2 megawatts.

It the single largest wind turbine with a rotor diameter of nearly $100 \mathrm{~m}$. [5] The first massive leap in the history of wind turbines happened during the Danish development in 1978 when the world's first multi-megawatt wind turbine power plant was set up. It delivered 2MW, had pitch controlled wings, a tubular tower, and three rotor blades. However, wind turbine could never enter into a near competition with crude oil and natural gas in producing power, until recently, that the fears of petroleum shortages added to urgency to expand wind power. By 2014, over 240,000 commercial-sized wind turbines were operating in the world, producing $4 \%$ of the world's electricity. By 2015, the largest wind turbine was 8 MW capacity Vestas V164 for offshore use. [6] Observing the recent (since quite some time) trend of development in wind turbines has chiefly been in the elongation and making slender of the rotor blades and heightening of the tower, to sum up. This has been guided by the simple relation: Power generation is directly proportional to size of rotor blades and height in the atmosphere (at higher altitudes in atmosphere, wind blows more steadily), leading to increase in turbine capacity factor [10]. Bigger they get, the less variable and more reliable they get and the easier they are to integrate into the grid. The average total height of an onshore U.S. turbine is $142 \mathrm{~m}$ (466 feet). The Haliade-X. (Blade diameter expected to be 351 feet long, longer than a football field), which will be the biggest, tallest and most powerful wind turbine ever, is expected to ship in 2021[11]. The department of Energy's 2016 Wind Technologies Market Report has shown that wind capacity factors have evolved over time. The average capacity factors among projects built during 2014 to 2016 was $42.5 \%$, that of those built during 2004 to 2011 was $32.1 \%$ and 1998 to 2001 was $25.4 \%$.

\section{Equations}

Viscous k-epsilon(2 Eqn) Model:

$$
\mu_{t}=\rho C_{\mu} \frac{k^{2}}{\epsilon}
$$


Second Order Upwind Momentum Equation.

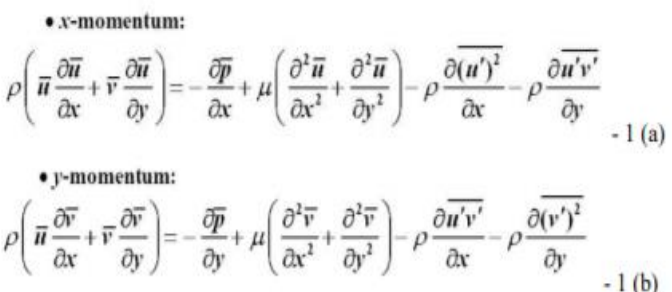

Second Order Upwind Turbulent Kinetic Energy Equation.

$$
\underbrace{\rho U_{i} \frac{\partial k}{\partial x_{i}}}_{\text {Convection }}=\underbrace{\mu_{t}\left(\frac{\partial U_{j}}{\partial x_{i}}+\frac{\partial U_{i}}{\partial x_{j}}\right) \frac{\partial U_{j}}{\partial x_{i}}}_{\text {Generation }}+\underbrace{\frac{\partial}{\partial x_{i}}\left\{\left(\mu_{t} / \sigma_{k}\right) \frac{\partial k}{\partial x_{i}}\right\}}_{\text {Diffusion }} \underbrace{-\rho \varepsilon}_{\text {Destruction }-2}
$$

Second Order Upwind Turbulent Dissipation Rate.

$$
\underbrace{\rho U_{i} \frac{\partial \varepsilon}{\partial x_{i}}}_{\text {Convection }}=\underbrace{C_{1 \varepsilon}\left(\frac{\varepsilon}{k}\right) \mu_{i}\left(\frac{\partial U_{i}}{\partial x_{i}}+\frac{\partial U_{i}}{\partial x_{j}}\right) \frac{\partial U_{i}}{\partial x_{i}}}_{\text {Generation }}+\underbrace{\frac{\partial}{\partial x_{i}}\left\{\left(\mu_{i} / \sigma_{i}\right) \frac{\partial \varepsilon}{\partial x_{i}}\right\}}_{\text {Diffusion }} \underbrace{-C_{2 \varepsilon} \rho\left(\frac{\varepsilon^{2}}{k}\right)}_{\text {Destruction }}
$$

$$
\begin{aligned}
& \sigma_{k,} \sigma_{\varepsilon}, C_{1 \varepsilon}, C_{2 \varepsilon}, C_{\mu} \quad \text { are empirical constants } . \\
& \sigma_{\varepsilon}=1.3 \\
& C_{\varepsilon 1}=1.44 \\
& C_{\varepsilon 2}=1.92\left[1-0.3 \exp \left(-R_{T}^{2}\right)\right]
\end{aligned}
$$

\section{Solution Methodology}

Initially, Solidworks Model of Whole Wind Turbine was framed and after that for Optimization of Wind Turbine Blades we designed Wind Turbine blades.

In the fig (1), incorporation of epoxy material and performed the Structural, Thermal analysis. In structural analysis, consideration of pressure of 151988 Pascal and fixed the central part of the design model and Force applied on Wind Turbine blades is 500 N. Body Sizing Meshing is followed for Meshing the Model. After that we obtained the following results:

1. Total deformation

2. Equivalent stress

3. Equivalent strain

After that Incorporation of fatigue analysis of wind turbine blades and after that Modal Analysis for performance check in Vibration using frequency mode obtained.

Frequency Mode for Fig (1):

Table 1:

\begin{tabular}{|l|l|}
\hline Mode & Frequency $(\mathrm{Hz})$ \\
\hline 1 & 451.79 \\
\hline 2 & 546.05 \\
\hline 3 & 2166.7 \\
\hline 4 & 3317 \\
\hline 5 & 4681 \\
\hline 6 & 5131.9 \\
\hline
\end{tabular}

After that Flow analysis is done for Wind turbine blades using standard parameter values:

Velocity incorporated: 83.33 m/sec, Pressure: 151988 Pascal, Temperature: $45 \mathrm{C}$ and Ambient Temperature: $40 \mathrm{C}$

Viscous k-epsilon model, Second Order Upwind Momentum Equation, Second Order Upwind Turbulent Kinetic Energy Equation and Second Order Upwind Turbulent Dissipation Rate equations have been incorporated for Computational Fluid Dynamics analysis.

In the Fig (2), also we had incorporated epoxy material and performed the Structural, Thermal analysis. In structural analysis, we have taken the pressure of 151988 Pascal and fixed the central part of the design model and Force applied on Wind Turbine blades is 500 N. Body Size Meshing is followed for Meshing the Model. After that we obtained the following results:

1. Total deformation

2. Equivalent stress

3. Equivalent strain

After that Incorporation of fatigue analysis of wind turbine blades and after that Modal analysis for performance check in Vibration using frequency mode obtained. Frequency Mode for Fig (2):

Table 2:

\begin{tabular}{|l|l|}
\hline Mode & Frequency $\mathbf{( H z )}$ \\
\hline 1 & 93.531 \\
\hline 2 & 606.44 \\
\hline 3 & 766.13 \\
\hline 4 & 1249.8 \\
\hline 5 & 1920.8 \\
\hline 6 & 3295.4 \\
\hline
\end{tabular}

After that Flow analysis is done for Wind turbine blades using standard parameter values:

Velocity incorporated: $83.33 \mathrm{~m} / \mathrm{sec}$

Pressure: 151988 Pascal

Temperature: 45 C

Ambient Temperature: $40 \mathrm{C}$

Viscous k-epsilon model, Second Order Upwind Momentum Equation, Second Order Upwind Turbulent Kinetic Energy Equation and Second Order Upwind Turbulent Dissipation Rate equations have been incorporated for Computational Fluid Dynamics analysis

After the Design and Simulation part, Incorporation of Simulink Model to enhance the performance of wind turbine to depict nature of Rotor Speed, Electromagnetic Torque and Stator Current.

Voltage: 3 x $480 \mathrm{~V}$

Track angle within 1 degree

Rise Time 3 seconds

\section{Figures and Tables}




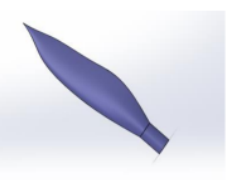

Fig- 1

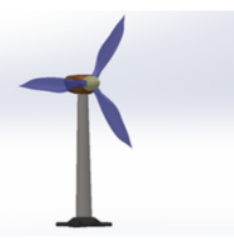

Fig-3

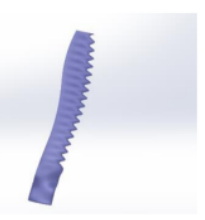

Fig-2

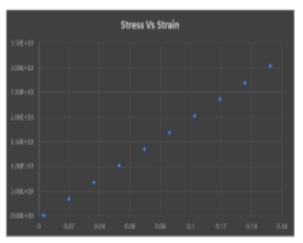

Fig-a

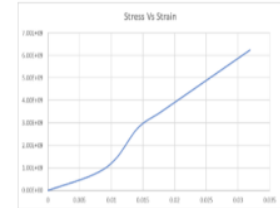

Fig-b Stress vs Strain Plot

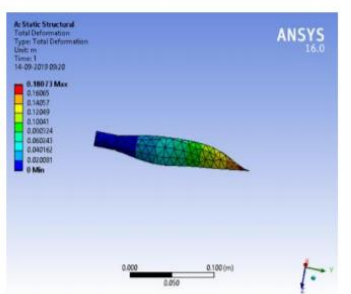

Fig-d

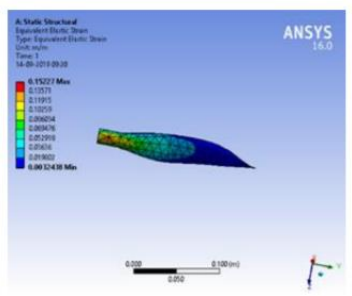

Fig-f

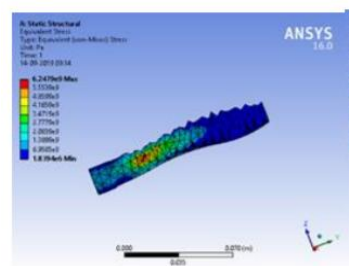

Fig-h

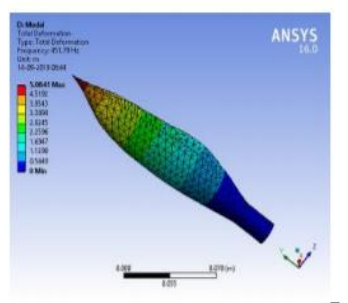

Fig-j

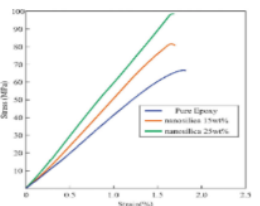

Fig-c

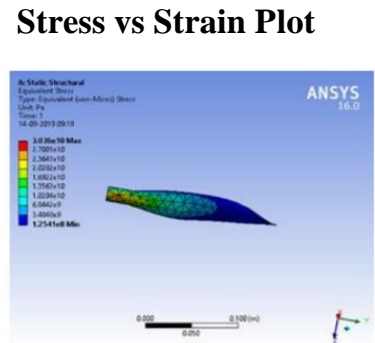

Fig-e

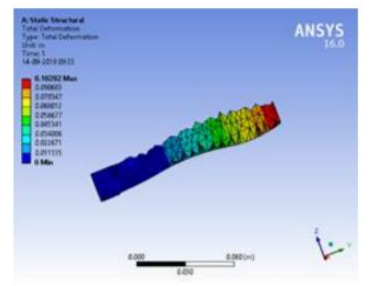

Fig-g

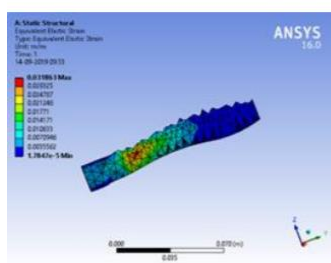

Fig-i

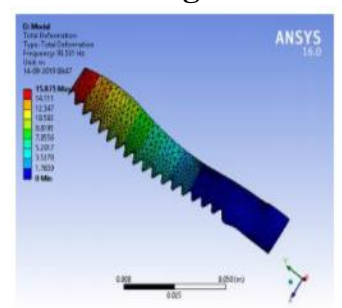

Fig-k

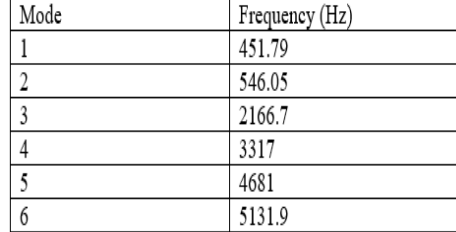

Table -1

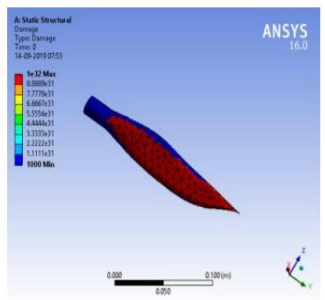

Fig-l

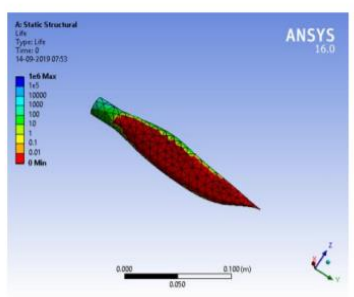

Fig-n

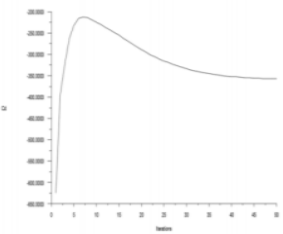

Fig-p

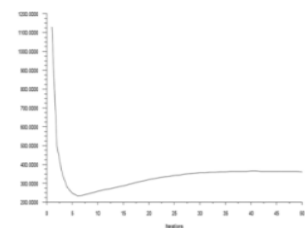

Fig-r

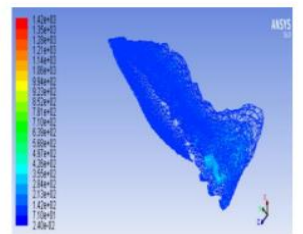

Fig-t

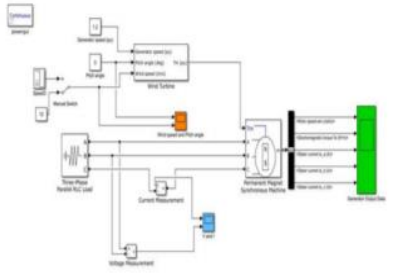

Fig-v

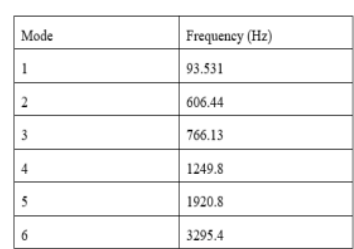

Table - 2

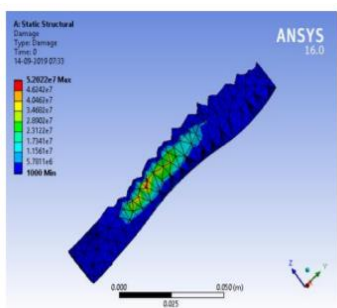

Fig-m

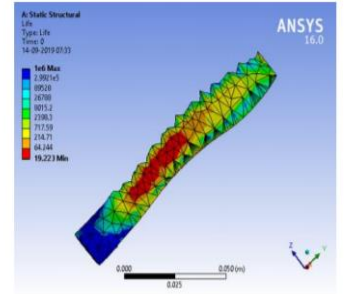

Fig-o

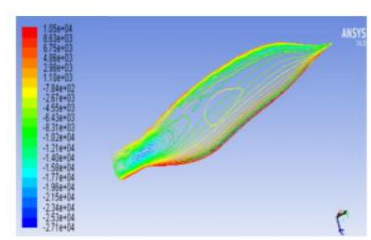

Fig-q

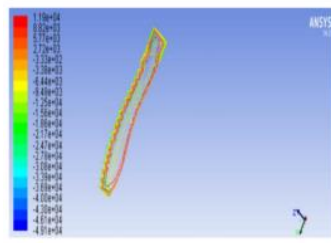

Fig-s

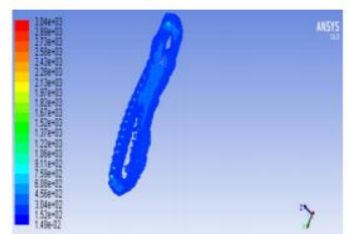

Fig-u

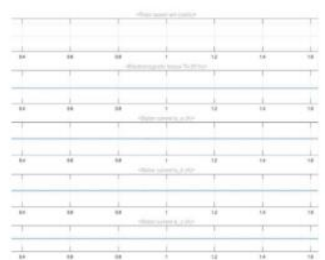

Fig-w

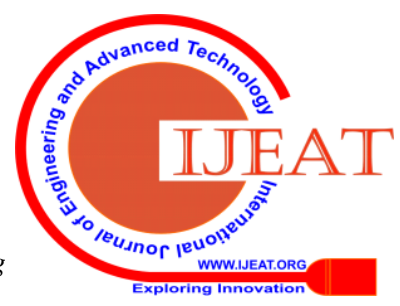




\section{RESULT AND DISCUSSION}

Validation of Stress vs Strain curve was done to validate the nature of Epoxy nature obtained from Design and Simulation. Fig(a), Fig(b) plots are compared with Fig(c) plot. It is observed Fig(b) is more similar to Fig(c) as compare to Fig(a).

Fig(d) and Fig(g) depicts the Deformation behavior in Fig (1) and Fig (2). Fig(e) and Fig(h) depicts the Stress Behavior in Fig (1) and Fig (2). Fig(f) and Fig(i) depicts the Strain Behavior in Fig (1) and Fig (2).

Deformation is less in Fig(g) as compared to Fig(d).

Equivalent Stress and Equivalent Strain Contour, Fig(e), Fig(f), Fig(g), Fig(h) clearly depicts the Stress Distribution and Strain Distribution in both the Wind Turbine blade profile. Behavior of Stress vs Strain Plot depicted by Fig(b) is more similar to Standard Epoxy Stress vs Strain Plot depicted by Fig(c). So, Efficiency of Fig (2) is more as compare to Fig (1) in terms of Structural Behavior.

Vibrational Frequency of Fig(k) is more optimized than Fig(j). So, Efficiency of Fig (2) is more improvised as compare to Fig (1). Frequency for particular mode observed is less in Table 2 as Compared to Table 1.

Fig(l) and Fig(m) depicts the damage profile for Fig (1) and Fig (2). Fig(n) and Fig(o) depicts the life profile for Fig (1) and Fig (2). Damage is more for Fig(l) as compared to Fig(m).

Life expectancy is more for Fig(o) as compared to Fig(n). So, Fig (2) is more Optimized.

Coefficient of Drag plot is observed in Fig(p) and Fig(r) for Depicting the Flow behaviour of Fig (1) and Fig (2).

Fig(q) and Fig(s) depicts the Flow Contour of Fig (1) and Fig (2). Drag along $x$ axis is 39.359135 and Lift along $Y$ axis is -2.2426147 obtained for Fig (2) is more appropriate as compare to Drag along $\mathrm{x}$ axis is 0.58842742 and Lift along $\mathrm{Y}$ axis is -2.7198 obtained for Fig (1). High Lift to Drag Parameter is always appropriate for Designing an efficient wind turbine blade. Since, Turbine blades are twisted so they can always present an angle that take advantages of the ideal lift-to-drag ratio. Coefficient of Drag plot depicted by Fig(p) is opposite to Coefficient of Drag plot depicted by Fig(r), Depicting the Variation of drag force with respect to different blade profile. Turbulence plot is observed for Fig(t) and Fig(u). Turbulence Enhancement is depicted in Fig(u) as compare to Fig(t) for overall depiction of performance characteristic of Fig (2) over Fig (1) in Turbulence. Behaviour depiction of Rotor Speed, Electromagnetic Torque and Stator Current was done by Simulink Model (i.e. Fig(v)) to optimize the performance of Wind Turbine Fig (3)

\section{CONCLUSION}

Efficiency of Wind Turbine is enhanced by optimizing the design of Wind Turbine blades. Performance Efficiency is achieved by reduction in Performance error. Proper Design Simulation was carried out to depict the behavior of turbine blades. Simulink Model was designed to optimize the performance of Wind Turbine. High Lift to Drag Parameter is optimized for proper Efficiency of Wind Turbine. Stress vs Strain curve validation was done to depict the nature of Epoxy nature obtained from Design and Simulation in both the models. Better energy Production parameter is solved by the analysis and Simulation. Optimization of Tower Design was carried out to enhance the performance of wind turbine. Flow Contour is depicted in Fig (q) and Fig(s). Efficiency of new Modelled blade design is more as compare to old modelled blade design.

\section{REFERENCES}

1. Small Wind Turbine augmentation: Experimental Investigation of shrouded and twin-rotor wind turbine systems.

2. Michal Lipian, Ivan Dobrev, Maciej Karczewski, Fawaz Massouh, Krzysztof Jozwik

3. Experimental Investigation of the Power performance of a minimal wind turbine array in an atmospheric boundary layer wind tunnel.

4. Bingzheng Dou, Michele Guala, Pan Zeng, Liping Lei

5. Pitch Angle Control of a wind turbine operating above the rated wind speed: A sliding mode control approach.

6. L.Colombo, M.L. Corradini, G. Ippoliti, G.Orlando

7. Experimental Validation of the power Enhancement of a pair of vertical axis wind turbines.

8. Antoine Vergaerde, Tim De Troyer, Lieven Standaert, Joanna Kluczewska-Bordier, Denis Pitance, Alexandre Immas, Frederic Silvret, Mark C. Runacres.

9. Hydraulically actuated horizontal axis wind turbine pitch control by model free adaptive controller.

10. P.Venkaiah, Bikash K. Sarkar

11. Trailing-edge serrations effect on the performance of a wind turbine.

12. Elena Llorente, Daniele Ragni

13. A comprehensive Review on Contemporary materials used for blades of wind Turbine.

14. A.V Pradeep, S.V. Satya Prasad, LV. Suryam, P. Prasanna Kumari

15. Fatigue Reliability assessment of offshoe wind turbines wind turbines with stochastic availability.

16. Jan-Tore Horn, Bernt J.Leira

17. Numerical Investigations into the idealized diurnal cycle of atmospheric boundary layer and its impact on wind turbines power performance.

18. Linlin Tian, Yilei Song, Ning Zhao, Wenzhong Shen, Tongguang Wang, Chunling Zhu

19. Numerical study for the flow field and power argumentation in a horizontal axis wind turbine.

20. Mona Abdelwaly, Hesham El-Batsh, Magdy Bassily Hanna

21. Effect of Geometric Uncertainties on the aerodynamics characteristics of Off shore Wind Turbine Blades.

22. Benedikt Ernst, Henning Schmitt, Jorg R.Seume

\section{AUTHORS PROFILE}

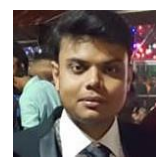

Bachelor of Technology degree in Mechanical Specialization in Automotive department from Vellore Institute of Technology and Automotive Research Association of India

Vehicle Dynamics Head \& Team Manager, KI-RACING (International BAJA Team).

Founder \& Team Captain, MOTO X (International Off road and Sports Cycle Team)

Student Organizer - Riviera 2020, (Annual Cultural Fest, VIT University, Vellore).

Student Representative for Indiana Summit 2019 at Indiana University Purdue University (IUPUI), Indianapolis, USA.

Student Representative of Automotive Branch in Automotive Research Association of India \& Vellore Institute of Technology, Vellore Publicity Chairperson for SAE-VIT (International Student Chapter). 